\title{
Real-time Planning for Construction Robots
}

\author{
B. Kahane and Y. Rosenfeld
}

\author{
Faculty of Civil Engineering and National Building Research Institute \\ Technion - Israel Institute of Technology \\ Haifa 32000, ISRAEL \\ Tel/fax. 972-4-8292363 \\ E-mail: roseny@tx.technion.ac.il
}

\begin{abstract}
Most past attempts to deal with the unique challenges facing mobile construction robots have involved tasks such as position finding, local mapping, and automatic calibration. It was found that the combination of numerous small errors, such as incorrect positioning of the robot's carriage or inaccuracy in interpreting the environment, with minor inaccuracies in the robot's arm movement, can result in errors of several millimeters. Thus, the robotic performance of high-precision or delicate tasks, such as in-situ tile setting or block laying, almost becomes a "mission impossible", unless real-time sensing and correction is applied for each and every element as it is set in place.

This paper presents a concept that enables an indoor mobile construction robot to be positioned in the close vicinity of its temporary workstation, with an approximate accuracy of about 10 centimeters. The robot then calibrates itself to the immediate environment, which is relevant only to the current workstation. The movements made by the robot's end-tool when bringing a building element (e.g. a tile) to its target position are directed in real-time by sensing the tool's immediate surroundings. The overall task cycle may start with a swift long-distance movement of the arm towards the target, followed by a series of fine, short-distance iterative "Sense-and-Act" cycles. This consistent interaction with the immediate environment renders unimportant many errors that may occur in the broader environment.

This general "Sense-and-Act" concept was applied and tested on one of the most challenging interior tasks for robots, namely accurate tile setting on walls. This task requires three-dimensional precision to the millimeter. A special device was developed for the delicate "Sense-and-Act" cycles of tile setting. The device consists of a CCD camera and several laser projectors, combined with sophisticated computer image processing. The device developed is introduced and discussed in the paper, along with a presentation of full-scale tile setting experiments.
\end{abstract}

KEYWORDS: CCD, Construction-robot, Laser, Real-time, Sense-and-act, Tile-setting.

\section{INTRODUCTION}

\subsection{Related construction robots}

Many studies conducted in the last two decades, sought a suitable way of introducing robotics into the field of construction.

Many research works suggested highly autonomous robotic systems for the performance of construction tasks, without fully investigating their implications. It is obvious that high levels of autonomy require the use of sophisticated sensory devices. Berlin (1994) described a robot designed for concrete surface processing, which receives the floor planning as an input, and after an automatic calculation of its moves, presents them to the operator for improvement before acting. Andres et al. (1994) suggested a masonry robot that pre-plans its tasks in detail. In spite of this, the robot introduced inaccuracies due to erroneous calibration, arm deformation, and so on. A complying end-tool was therefore suggested, which was designed to compensate for such inaccuracies. Chamberlain et al. (1994) conducted experiments with a masonry robot that relied on detailed planning but also required sensory devices for accurate interaction with its environment. The researchers noted the advantage of not needing to know the robot's absolute position. Pritschow et al. (1996) also dealt with a masonry robot designed to operate in an autonomous manner. This robot, however, could not assure the accuracy of every movement without the use of auxiliary devices and sensors.

Some researchers attempted to increase the autonomy level of robots by enabling them to 
map their environments and independently navigate through them. The mapping and navigation methods should then be adapted to construction sites, which are characterized by inaccurate geometries, numerous obstacles, and so on. Such navigation methods are expected to be able to deal with these difficulties, and succeed in achieving accurate enough results. Forsberg et al. (1997) suggested a robot for plastering that uses a rotational laser beam to measure and map its surroundings (walls and openings). The mapping data should be translated into a working plan, which would be presented to the operator for improvements. The suggested system depends on accurate navigation methods, should bring the robot to within $\pm 1 \mathrm{~cm}$ of its workstation, a feat that appears to be unattainable.

Beliveau et al. (1996) developed an orientation system for indoor automated guided vehicles (AGVs), using three laser transmitters accurately positioned on the floor at known points. Experiments with this system revealed that the deviation of the measured path from the desired path was $\pm 10 \mathrm{~cm}$.

Shohet and Rosenfeld (1997) examined the achievable accuracy of automatic mapping of indoor construction environments. It was found that under conditions of precise robot positioning (orientation and location errors of $0.2^{\circ}$ and $3 \mathrm{~cm}$, respectively), the achievable accuracy of environment mapping is $3-5 \mathrm{~cm}$. This accuracy is sufficient for tasks that do not require touching the treated element (e.g. spraying); however, tasks that involve precise placing of elements (e.g. block laying and tiling), require a mapping accuracy of 2-3 $\mathrm{mm}$, as well as the utilization of well-controlled end-tools. The study concluded that this level of accuracy cannot be achieved at construction sites.

\subsection{Related robots in other fields}

Real-time planning is commonly employed in robotic tasks that are required to contend with uncertainties and undefined environments, yet must be performed accurately.

Tillett et al. (1995) claimed that the use of accurate robots for agricultural tasks is unjustified, and that it would be more suitable to use less accurate robots that are guided by a vision system. This approach was implemented in a robotic system for packing tomatoes. A camera identified tomatoes on a tray and guided the end-tool in grabbing them one-by-one using a suction device.

Sevila and Baylou (1991) characterized agricultural robotic work. Some of the characteristics described resemble those of robotized construction: Identifying various types of objects in natural environment conditions; interactivity between sensors and end-tools; working under undefined and sometimes hostile conditions; suiting the robot configuration to changing surface conditions, and so on. The authors noted that vision systems are best suited for the identification of a range of sizes, shapes, and colors. Yet, in order to simplify the vision analysis it was suggested to add and integrate other sensors as well. For example, an automatic milking system was constructed that recognizes udders by projecting a laser beam on them, the marks of which were detected by a camera. Different projection angles enabled the construction of a 3D representation of the udders (Figure 1).

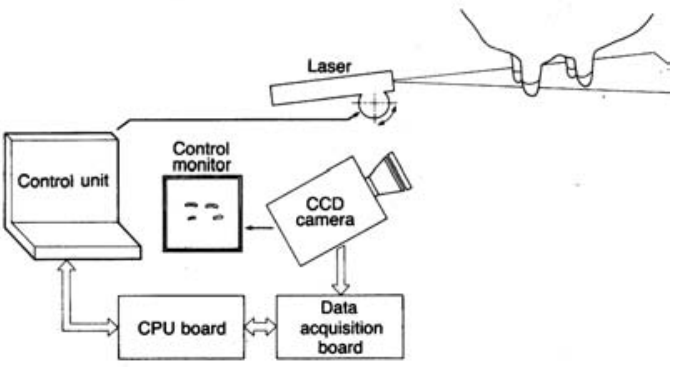

Figure 1. Udder detection (Sevila \& Baylou, 1991).

Another example of real-time planning was a robot vehicle that identifies asparagus plants on a furrow by means of a camera and a light projector (figure 2).

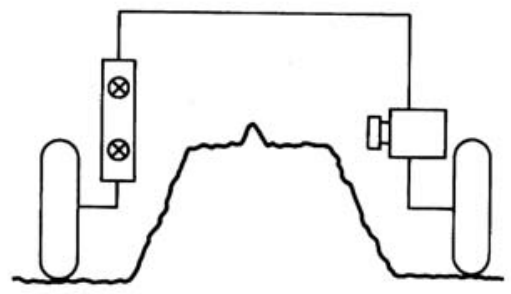

Figure 2. Plants detection (Sevila \& Baylou, 1991).

Sevila and Baylou clarified that a main problem in robotized agriculture is the difficulty in calibrating the system in relation to its environment. The common devices used for 
environment detection are not accurate enough for the performance of such tasks; hence, the use of sensory devices for detecting the immediate environment of a treated element is required.

A different field in which real-time planning can be demonstrated is welding by robots. In such tasks, the robot identifies the seam to be welded and tracks the seam while welding it.

A well-known method for seam tracking utilizes a camera and a laser beam. In this method, a laser beam is projected on the seam and a CCD camera identifies the broken line, which is indicative of the location of the seam (Figure 3 ).

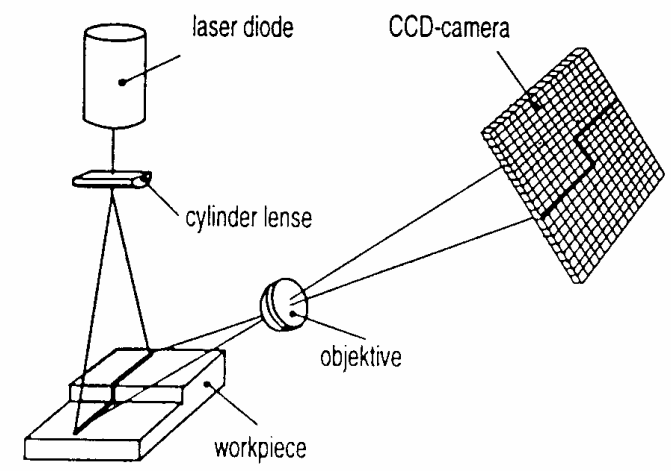

Figure 3. Seam detection using a laser and a camera (Haug and Pritschow, 1998).

\subsection{Problem definition}

Initially, robots were developed for the manufacturing industry and were intended to perform routine tasks in a very familiar environment, while fixed to the floor. Unlike such robots, those designated for work on construction sites must be mobile, maneuver in changing environments, and perform a different task at almost every step. Researchers and developers of autonomous robots for indoor construction tasks have attempted to solve the problem of adjusting the robot to its environment by developing automatic mapping and self-positioning methods. The robot then autonomously navigates from one workstation to another. Yet, it is clear that these methods are not likely to conform to the accuracy requirements of many construction tasks. In most cases, the robot must be accurately positioned at its workstation. Moreover, even when the correct positioning of the robot is assumed, accuracy of the robot's arm, or even that of the environment's interpretation, is not sufficiently reliable. The accuracy of the manipulator's moves may differ in each cycle due to variations in the load at the arm's end and in the arm configuration. Creating a more robust arm, one that would be less sensitive to the varied loads and trajectories, would lead to heavier and more expensive robotic systems with lower economic feasibility.

The drawbacks of the existing work methods are manifested most clearly when performing element-setting tasks, such as block laying and tile setting. In the latter case, it is essential to set each tile on the correct wall-plane, and to maintain the correct distance from other tiles set earlier as part of either the current workstation or previous workstations. Every visible error will adversely affect the outcome, or worse - could cause overlapping when trying to set one tile on top of another.

\section{THE SUGGESTED CONCEPT}

The concept presented herein suggests that the robot merely be positioned (or selfpositioned) at its workstation with an approximate accuracy of several centimeters. After positioning is completed, the robot will be calibrated relative to its immediate environment (the environment relevant to the workstation), and according to the robot's actual position, the system will plan (or replan) the appropriate manipulator moves. Moreover, the movement of the robot's end-tool to its position will be directed in real-time by sensing the tool's immediate surroundings. In other words, for each manipulator move used in the placing of an element, the system will ascertain the correct positioning of the element by performing several "Sense-and-Act" cycles. This consistent interaction with the immediate environment will eliminate many errors that may occur in a broader environment, such as location mistakes, mapping errors, manipulator deflection, and so on.

Three main steps are performed after stabilization of the robot at the workstation, as follows:

Rough calibration - After stabilizing the robot at its workstation (manually or autonomously), the robot will sense and determine its position relative to its environment. The robot will recognize the wall-plane, the boundaries of the present work section, and the boundaries of the neighboring work sections. Special elements in the work section (e.g. openings in the wall) will also be recognized at this stage. Other complimentary data sources, such as general information and local 
data, can support the calibration process by adding relevant information about expected measurements and positions.

Planning the task at the workstation - According to the calibration data, the robot can relate a work plan to the actual environment, and roughly plan the relevant moves of the manipulator for the current work section. Before executing its task actions, the robot will present the work plan to the operator, who will examine it, correct it if necessary, and approve its execution.

Fine calibration - While executing the task, fine calibration will be performed during each cycle of manipulator moves aimed at placing an element. During the calibration cycles, sensors will collect accurate data on the end-tool's immediate environment. Based on these data, the robot will update the manipulator trajectory and move the element closer to its target position. In this iterative "Sense-and-Act" process, the sensing range will be gradually reduced, thus increasing the accuracy of the data collected. As a result, the manipulator moves for setting of the element will be more accurate.

\section{PRINCIPLES OF THE METHOD}

The suggested method is based on the three following principles:

Close reference planning - Planning of manipulator movements at a specific workstation is performed only after the robot is stabilized in its position. This means that there is no need to have advance knowledge of the surroundings' details nor is there any need for an accurate navigation system. Moreover, there is no need to prepare the building design as a basis for planning the robotic work. It is helpful, though, to use the building plans as guidelines in order to identify repeated elements, thus facilitating the definition of the workstation environment.

Local calibration - A focus is placed on the calibration of the robot in relation to its close environment, after stabilizing at the workstation. This principle enables the neutralization of the effect of stabilizing errors on the accuracy of the task performance. This type of calibration must integrate various types of sensors for short-range recognition of the robot's surroundings, in a satisfactorily accurate and detailed manner.
Continuity - The performance of each work cycle is derived from the performance of the preceding cycles, i.e., when setting a tile to a wall, its exact position will be realized relative to the position of the tiles already set, and not based solely on precalculated coordinates. To achieve this, special sensory devices must be developed and specially adapted to each task.

\section{TILE SETTING END-TOOL}

The heart of the "Sense-and-Act" method is the "fine calibration" stage, which provides the expected accuracy required for the task. Development of a sensory device, which will be appropriate for the end-tool's accurate final moves, must be customized to the specific characteristics of the task at hand. Due to its extreme accuracy requirements, tile setting is one of the most complex construction tasks that can be performed by robots.

The suggested tool consists of a suction gripper, a CCD camera, and five laser line projectors. The CCD camera is mounted, as part of the end-tool, in such a way that it views the corner of the gripped tile. Each laser projector projects its light in an oblique way, as described in Figure 4.

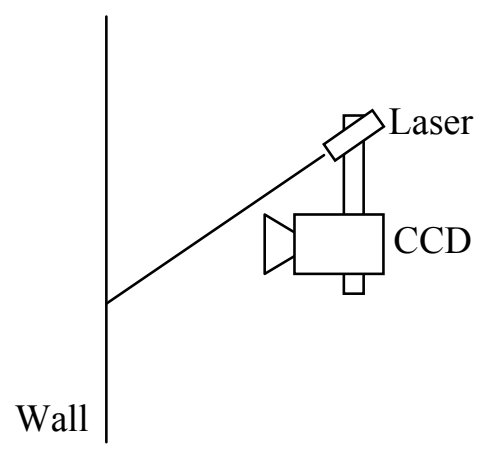

Figure 4. A single laser beam relative to the $C C D$ camera.

Projecting the laser line over the joint between two tiles produces an image of a broken line, as demonstrated in Figure 5. 


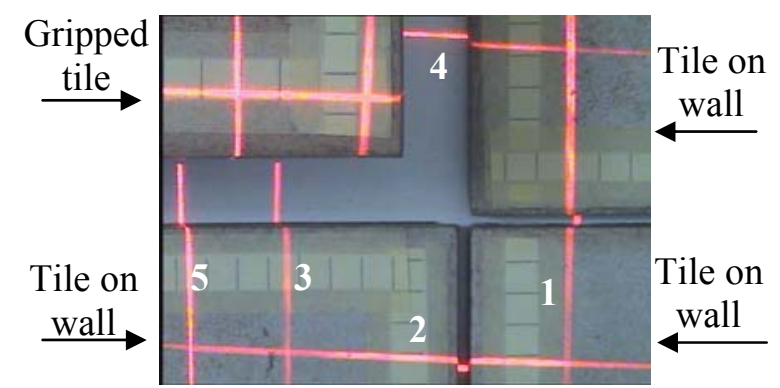

Figure 5. The revealed image of 5 projected laser lines.

The proposed sensing system identifies the broken section of each line (method not described here) and measures its length in pixel units (denoted $\Delta i$ where $i=1 \ldots 5$ in Figure 5). The system then calculates the differences between the breaks for several specific pairs of lines. The deviations in the position of the gripped tile are proportional to the following differences:

"Angular deviation" $\propto(\Delta 5-\Delta 3)$

"Horizontal deviation" $\propto(\Delta 3-\Delta 1)$

"Vertical deviation" $\propto(\Delta 4-\Delta 2)$

After calculating the above deviations, the system commands the robot to correct them. This cycle of "Sense-and-Act" is repeated several times; each time, the gripped tile is moved closer and closer to its final position on the wall.

This sensing method is suitable for the typical situation of setting a new tile adjacent to three other, already set, tiles. The boundary cases, i.e. the first row and first column of tiles, must be solved manually. The robot operator will then set the first row and the first column of tiles as guidelines for the robot.

\section{EXPERIMENTS}

Full-scale laboratory testing was performed on the proposed sensing method (Figure 6).

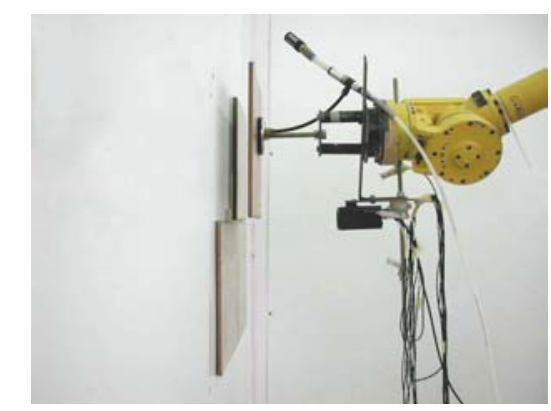

Figure 6. The experimental end-tool.
An end-tool was built and tested in order to determine its ability to accurately detect deviations. The correction of linear deviations was tested separately from the correction of angular deviations. For calibration purposes, a gripped tile was set in its final position on the wall with negligible errors, and at a distance of $3 \mathrm{~mm}$ from its neighboring tiles. Then, the tile was positioned at a starting point located $60 \mathrm{~mm}$ from the wall, with known deviations of $20 \mathrm{~mm}$ in the linear case and $3^{\circ}$ in the angular case. Several scenarios were tested that differed in the number of iterations and closing ranges performed until setting of tile in its final position. The resulting error was calculated by comparing the actual final position of the gripped tile with the calibrated final position.

The experiments revealed that:

- The attainable error for the linear correction was $0.2 \mathrm{~mm}$. This error is perfectly acceptable.

- The attainable error for the angular correction was $0.3^{\circ}$. This error seems to be unacceptable.

Further improvements of the end-tool, based on an additional CCD camera and laser projector, can improve the angular accuracy to an acceptable error of $0.1^{\circ}$. Moreover, the same tool can also measure its own distance from the wall with an error of merely $0.5 \mathrm{~mm}$.

\section{CONCLUSIONS}

Planning and executing the arm movements of a construction robot based on the building design is a most technologically demanding process. In contrast, a robotic system that would operate with no need for detailed preplanning would be less technologically demanding and may, therefore, be easily developed during the early stages of the integration of robotics into the construction field. The suggested "Sense-and-Act" process eliminates the need for high accuracy when positioning the robot in its workstation, a fact that saves time and leads to a greater economic feasibility of the system. The "Senseand-Act" scenario is simple and may indeed become a reality, as a midway stage in the development of more advanced robotic systems in construction.

An essential component of the "Senseand-Act" method is an end-tool that is able to accurately perform the "fine calibration" stage. This paper presented the basics of such an endtool designed for the task of tile setting, and, 
following its satisfactory performance, demonstrated the feasibility of the "Sense-andAct" method.

\section{REFERENCES}

Andres J., Bock T., Gebhart F. and Steck W. (1994), First results of development of the masonry system ROCCO: A fault tolerant assembly tool, Proceedings of The 11th International Symposium on Automation and Robotics in Construction (ISARC), Brighton, U.K., 87-93

Beliveau Y.J., Fithian J.E. and Deisenorth M.P. (1996), Autonomous vehicle navigation with real-time 3D Laser based positioning for construction, Automation in Construction, 5(4), 261-272.

Berlin R. (1994), Development of a multi-purpose mobile robot for concrete surface processing, Proceedings of the 11th International Symposium on Automation and Robotics in Construction (ISARC), Brighton, U.K., 133140.

Chamberlain D.A., Akrawi S. and Waston J. (1994), Path planning and sensing for an experimental masonry building robot, Automation in Construction, 3(1), 65-70.

Forsberg J., Aarenstrup R. and Wernersson A. (1997), A construction robot for autonomous plastering wall and ceilings, Proceedings of the 14th International Symposium on Automation and Robotics in Construction (ISARC), Pittsburgh, Pennsylvania, U.S.A., 260-268

Haug K. and Pritschow G. (1998), Robust Laserstripe Sensor for Automated Weld-seamtracking in the Shipbuilding Industry, Proceedings of the 24th Annual Conference of the IEEE Industrial Electronics Society (IECON), Aachen, Germany, 2, 1236-1241.

Pritschow G., Dalacker M., Kurz J. and Gaenssle M. (1996), Technological aspects in the development of a mobile bricklaying robot, Automation in Construction, 5(1), 3-13.

Sevila F. and Baylou P. (1991), The principles of robotics in agriculture and horticulture, Progress in Agricultural Physics and Engineering, Matthews J. (ed.), 119-147.

Shohet I.M. and Rosenfeld Y. (1997), Robotic mapping of building interior - precision analysis, Automation in Construction, 7(1), 112

Tillet N.D., He W. and Tillet R.D. (1995), Development of a vision guided robot manipulator for packing horticultural produce, Journal of Agricultural Engineering Research, 61(3), 145-154. 